\title{
Sleep bruxism. Conceptual review and update
}

\author{
José-Luis de la Hoz-Aizpurua ${ }^{1}$, Esperanza Díaz-Alonso ${ }^{2}$, Roy LaTouche-Arbizu ${ }^{3}$, Juan Mesa-Jiménez ${ }^{4}$
}

${ }^{1} \mathrm{MD}, \mathrm{DMD}, \mathrm{MS}$, Coordinator and Professor

${ }^{2}$ Physical Therapist

${ }^{3}$ Physical Therapist, Coordinator and Professor

${ }^{4}$ Physical Therapist, Director and Professor

Correspondence:

Master's Degree Program in Craniomandibular

Dysorders and Orofacial Pain

Universidad San Pablo CEU,

Madrid, Spain

jhoz@infomed.es

Received: $23 / 05 / 2010$

Accepted: $28 / 05 / 2010$

\author{
de la Hoz-Aizpurua JL, Díaz-Alonso E, LaTouche-Arbizu R, Mesa-Jimé- \\ nez J. Sleep bruxism. Conceptual review and update. Med Oral Patol Oral \\ Cir Bucal. 2011 Mar 1;16 (2):e231-8.
}

http://www.medicinaoral.com/medoralfree01/v16i2/medoralv16i2p231.pdf

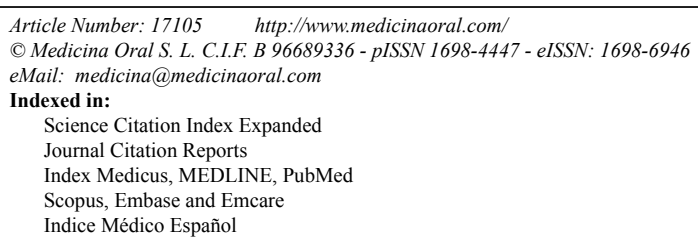

Indice Médico Español

\begin{abstract}
Sleep bruxism (SB) is a parafunctional oromotor habit that can sometimes pose a threat to the integrity of the structures of the masticatory system if the magnitude and direction of the forces exerted exceed the system's adaptive capacity.

Over the years science has tried to provide a consistent explanation of the etiopathogenesis and physiopathology of SB, although the pathophysiological mechanisms are even now not yet fully understood.

There is at present no specific, effective treatment to eliminate the habit of bruxism permanently. There are only palliative therapeutic alternatives steered at preventing the pathological effects of SB on the stomatognathic system and alleviating the negative clinical consequences of the habit.

The objective of this article is to review and update the fundamental scientific concepts of SB and to furnish an approach to the main types of therapy used, based on the scientific literature.
\end{abstract}

Key words: Bruxism, sleep bruxism, oral dyskinesis, oromotor disorders, occlusal splint.

\section{Concept}

Sleep bruxism (SB) is a parafunctional oromotor habit that can sometimes pose a threat to the integrity of the structures of the stomatognathic system if the magnitude and direction of the forces exerted exceed the system's adaptive capacity.

In the second International Classification of Sleep Disorders (AASM 2005), SB is defined as an oromotor activity that is characterised by the clenching or grinding of the teeth during sleep, is habitually associated with micro-arousals and is generally accompanied by sound.
This definition has changed since the first edition of the International Classification, in which SB was classified amongst the parasomnias (abnormal behaviour presenting during but not interrupting sleep).

Kato and cols. affirm that SB is a parasomnia and a parafunctional activity during sleep that is characterised by clenching (tonic activity) and/or the repetition of phases of muscle activity (phasic activity) that produce grinding of the teeth (1).

The American Academy of Orofacial Pain in 2008 defined bruxism as a diurnal or nocturnal parafunctional 
activity that includes unconscious clenching, grinding or bracing of the teeth. Bruxism can be suspected when there is clear evidence of wear facets that cannot be interpreted as the result of the masticatory function. The presence of SB can be confirmed by polysomnographic recording in a sleep laboratory. This definition is somewhat less precise, since it uses the terms "diurnal" and "nocturnal" instead of "sleep" and "waking", which are more in accordance with the dynamics of life in today's society (not all people sleep at night and "live" during the day).

\section{Epidemiology}

Sleep bruxism is a frequent habit. It is present, at least episodically, in a significant percentage of the population. Nevertheless, the prevalence of SB is difficult to estimate because, in most cases, there are no clinical symptoms. In those cases where there is some clinical manifestation, bruxism is initially suspected due to the presence of symptoms reported in anamnesis by the patient or the patient's bed partner or due to the presence of suspicious clinical signs.

Only polysomnography (PSG) in a sleep laboratory can confirm a definite diagnosis, and being PSG a very costly test, make the possibility of conducting cross-sectional studies amongst the population difficult. In addition, in the interpretation of a polysomnogram, there are many recordings of movements of the masticatory and orofacial musculature (e.g., swallowing, tics, coughing) that may prove confusing. This point will be dealt with in greater detail in the discussion of diagnosis.

Furthermore it must be kept in mind that PSG studies are generally prescribed as complementary testing for the diagnosis of clinical entities that may be associated with SB and whose treatment may also alter the results of the study.

The two most-representative large-scale epidemiological studies conducted by telephone survey coincide in estimating an approximate SB prevalence of $8 \%$ in the general population (2). The prevalence of SB in children is between 14 and $20 \%$, in young persons between the age of 18 and 29 it is $13 \%$, in adults it is $9 \%$ and in persons over age 65 it is $3 \%(3-5)$.

\section{Etiopathogenesis}

The etiopathogenesis of SB continues to be the grounds for scientific debate. Different theories have been posited in succession over the years.

Although initially SB was thought to originate as a consequence of alterations in dental occlusion, the scientific literature has repeatedly proved that this theory lacks scientific validity (6).

At present studies seem to support the multi-factorial hypothesis of the genesis and pathogenesis of SB. Factors such as genetics (3), sleep structure (micro-arous- als) (7), environment, emotional distress, anxiety and other psychological factors (8), the catecholaminergic balance of the CNS (9), the autonomic nervous system, and some drugs (ecstasy, alcohol, caffeine, tobacco) and medications (e.g., selective serotonin reuptake inhibitors, benzodiazepines, dopaminergic drugs) bear a relationship with the etiopathogenesis of SB.

The theories that sustain a possible genetic predisposition to SB stem mostly from studies based on questionnaires or analyses of populations of monozygotic twins $(3,4)$. These studies reveal that between 20 and $50 \%$ of the individuals aware that they have SB have a direct relative who also has SB. This relationship is especially significant in monozygotic twins (4). Future research studies are oriented toward discovering the genes and proteins whose expression may bear a relationship with the genesis of SB.

Anxiety, emotional distress, their relationship with the hypothalamic/hypophyseal axis (release of catecholamines such as norepinephrine, epinephrine and dopamine) and their possible involvement in the etiopathogenesis of SB have also been the object of scientific debate (8).

High levels of catecholamines have been detected in the urine of children and adults with SB and have been related with stress levels. Also the results of a questionnaire-based study suggest that patients with SB have a deficit in their capacity to adapt and react to situations of stress (8).

However, one study using EMG was unable to prove a significant association between stress and SB. Only $8 \%$ of the study subjects displayed a relationship between stressful daytime situations and SB.

Another hypothesis that attempts to explain the etiopathogenesis of SB is based on cerebral neurotransmitters and their control over sleep/waking cycles (7, 9), stress response and autonomic activity. Substances such as epinephrine, norepinephrine, dopamine, serotonin and $\gamma$-aminobutyric acid (GABA) may be related with the genesis of rhythmic masticatory muscle activity (RMMA), although the scientific evidence is still weak.

It has been suggested that dopamine in the basal ganglia, which control mobility of the mouth, and in the limbic system, which is related with the processing of affections and emotions, may be of some importance in the origin of SB (10). The hypothesis is that there is an asymmetry in the dopamine uptake level of the D2 receptors situated in the said nuclei. This asymmetry could favour the appearance of SB in stressful situations that stimulate the production and secretion of dopamine in the substantia nigra of the midbrain (mesocorticolimbic and nigrostriatal pathways). However, other studies fail to confirm this hypothesis $(11,12)$. In fact, L-dopa, a dopamine precursor, has only a modest effect on the 
reduction of $\mathrm{SB}$, and bromocriptine, a dopamine agonist, has none (13).

Nor have any results been obtained that scientifically support the idea that serotonin plays a role in the etiopathogenesis of SB. Its precursor, tryptophan, and the use of tricyclic antidepressants that regulate serotonin levels in the CNS do not significantly affect the presence of SB.

The importance of the interaction amongst the noradrenergic, cholinergic and GABAergic neurons in the regulation of muscle tone in the different phases of sleep is known (14), as is the role that norepinephrine plays in maintaining alertness and wakefulness in situations of stress. The influence of norepinephrine on the etiopathogenesis of SB is supported by studies that show the action of clonidine, an $\alpha 2$-adrenergic agonist, in the reduction of the frequency of RMMA movements, with the drawback of producing severe orthostatic hypotension in $20 \%$ of the patients in the study.

Lastly, GABA is the primary CNS-inhibiting neurotransmitter. Although the hypothesis has been formulated that GABA may play a role in $\mathrm{SB}$, any relationship seems to be merely indirect, since GABA is implicated in almost all the neuronal systems that control wakefulness, sleep and motor activity. Its best-known pharmacological analogues, diazepam and clonazepam, produce a reduction of SB, but they also have severe, undesirable collateral effects, such as sleepiness, dizziness and risk of addiction (15).

According to the concepts explained above, SB may be divided into primary or idiopathic $\mathrm{SB}$, that is present without any apparent cause, and secondary SB, due to traumatic injuries, diseases or sometimes pharmacological treatments for other diseases, such as Parkinson's disease (iatrogenic bruxism).

\section{Physiopathology}

Sleep bruxism is related with RMMA $(11,12)$, a specific type of masticatory muscle activity that is characterised by rhythmic, pseudo-masticatory jaw movements occurring once or twice per hour of sleep, at a frequency of approximately $1 \mathrm{~Hz}$, especially in phases 1 and 2 of non-REM sleep. This masticatory parafunctional activity is observed in $60 \%$ of non-bruxist individuals and $80 \%$ of patients with SB. Although it is suspected that RMMA may be associated with increases in salivation to lubricate the oropharyngeal structures or to increase the space of the upper airways, its physiopathological relationship with SB has yet to be clarified, because RMMA is also present in other sleep disorders, such as parasomnias, acid reflux during sleep, obstructive sleep apnoea and snoring.

Although at present the nuclei and mechanisms that generate RMMA are not accurately known, it is thought that they may in some way be related with the central pattern generator, a complex framework of centres situated in the frontal zone of the nucleus of the trigeminal nerve that is responsible for controlling rhythmic masticatory movements during wakefulness, together with other structures of the encephalic trunk (motor nuclei of the trigeminal and facial nerves, lateral zone of the reticular formation, caudal pontine nucleus, principal sensory nucleus) $(14,16)$.

The physiopathology of SB seems to bear some relationship with an activation of the autonomic nervous system, specifically the sympathetic nervous system.

In fact, as shown in figure I, the oromotor episode of $\mathrm{SB}$ is only the final element in a chain of events that begins with sympathetic autonomic activation and a reduction of parasympathetic activity (between 8 and 4 minutes prior to the bruxing episode), followed by cortical activation with the presence of $\alpha$ waves on the EEG (4 seconds prior), an increase in respiratory and cardiac frequency (1 second prior) and an increase in the tone of the mouth-opening suprahyoid muscles $(0.8$ seconds prior), and ends with the bruxing episode (1, 11). This confirms the etiopathogenic hypothesis of the central origin of SB, where masticatory muscle activity, and therefore the contact of clenching or grinding the teeth, are only the peripheral reflection of this central activation, and it invalidates occlusal theories about the bruxing habit and questions the occlusal odontological therapeutic approach.

Close to $80 \%$ of the episodes of bruxism appear in groups or clusters during the transition from phase 3 to phase 2 and from phase 2 to phase 1 of non-REM sleep, in the passage from deep sleep to superficial sleep associated with micro-arousals (short-lasting three- to 15 -second periods in which there is a cortical activation associated with an increase in the activity of the sympathetic nervous system) $(11,14)$. These micro-arousals are physiological episodes that recur during sleep, grouped cyclically into what is known as the cyclic alternating pattern.

Sleep bruxism is observed more frequently during REM sleep in patients with psychiatric and/or neurological disorders and in patients being treated with drugs that act on the CNS $(9,10,13)$. To date polysomnography studies have revealed that there is a relationship between micro-arousals (autonomic cortical activation preceding the activation of the musculature that closes the mandible) and episodes of sleep bruxism, suggesting that the activation of the autonomic and central nervous systems is the primary factor responsible for the initiation of SB $(1,11,12)$. Even so, it should be stressed that most bruxist patients do not report poor sleep quality or present alterations in polysomnography studies $(7,11,12)$.

It was recently seen that close to $60 \%$ of the episodes of RMMA in patients with and without SB occurred 


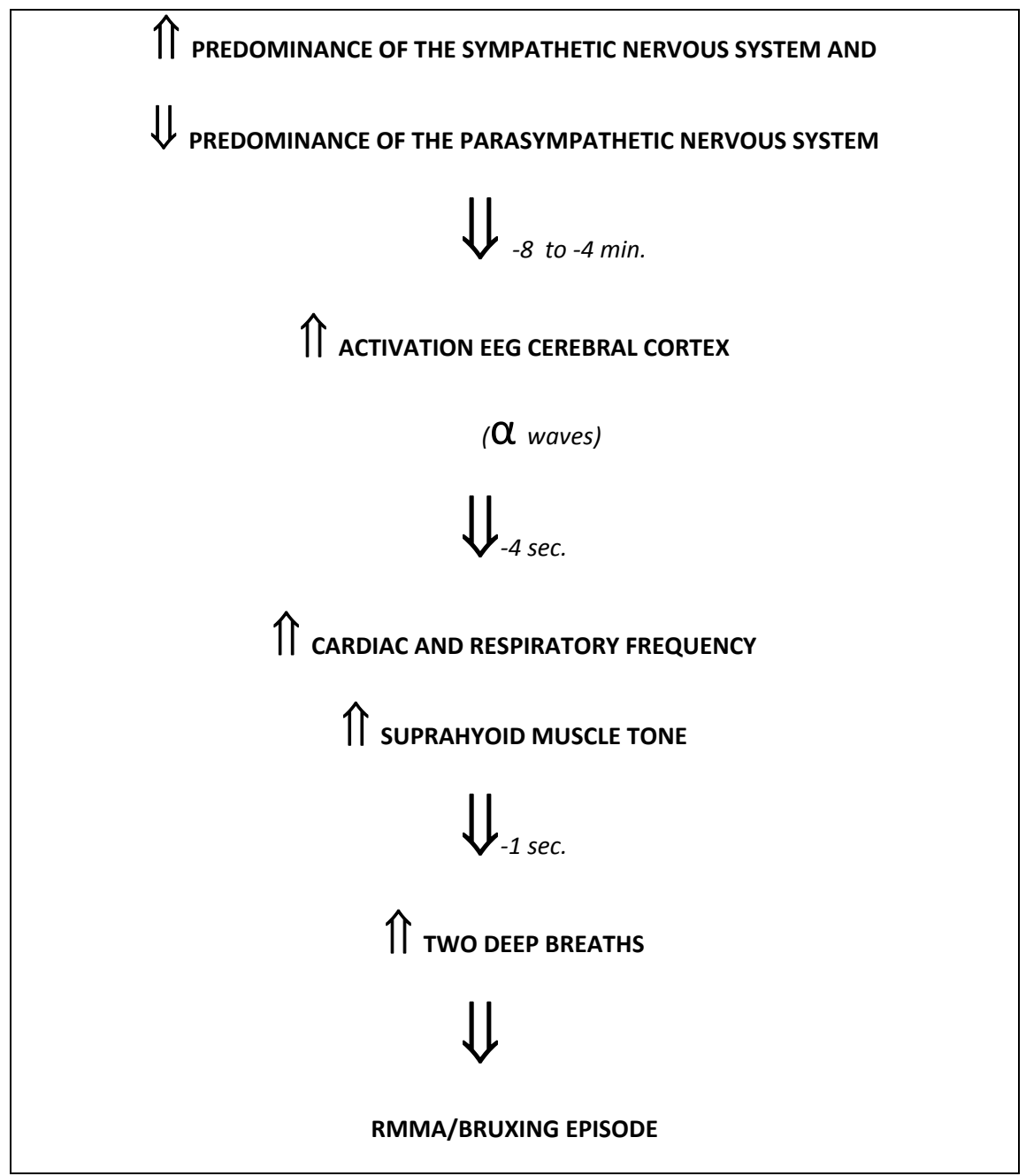

Fig. 1. Time sequence of physiological phenomena of micro-arousal with RMMA preceding an episode of SB.

concomitantly with laryngeal movements related with swallowing, and in addition nearly $80 \%$ of the episodes of SB were associated with leg movements $(1,2,12,17$, 18).

\section{Classification}

Bruxism may be classified according to several criteria (11):

1. By when it occurs:

a. Awake bruxism: This is presented when the individual is awake.

b. Sleep bruxism (SB): This is presented when the individual is asleep.

c. Combined bruxism: This is presented in both situations.

2. By aetiology:

a. Primary, essential or idiopathic bruxism: For which no apparent cause is known.

b. Secondary bruxism: Secondary to diseases (coma, ictus, cerebral palsy), medicinal products (e.g., antipsychotic medication, cardioactive medication), drugs (e.g., amphetamines, cocaine, ecstasy).

3. By motor activity type:

a. Tonic: Muscular contraction sustained for more than two seconds.

b. Phasic: Brief, repeated contractions of the masticatory musculature with three or more consecutive bursts of electromyographic activity that last between 0.25 and two seconds apiece.

c. Combined: Alternating appearance of tonic and phasic episodes.

Approximately $90 \%$ of the episodes of SB are phasic or combined, unlike in awake bruxism, where episodes are predominantly tonic.

4. By current or past presence:

a. Past bruxism.

b. Current or present bruxism.

They are frequently difficult to tell apart. 


\section{Clinical Picture}

Although SB is a parafunctional oromotor activity in which extremely intense force vectors are sometimes developed, most bruxers present no pathological clinical signs or symptoms (1).

In those cases where the bruxist parafunctional forces exceed the tolerance threshold of the masticatory system, the functional equilibrium of the stomatognathic system may be altered, at which time clinical symptomatology and signs of functional limitation appear, as shown in table 1 . These signs and symptoms may give rise to a picture of craniomandibular disorder (19).

\section{Diagnosis}

Clinical diagnosis on a suspicion of SB is conducted in patients who present with symptoms such as those described in table 1 and in whom signs compatible with the bruxing habit are found. This presumed clinical di- agnosis must be confirmed by instrumental diagnostic testing (PSG, EMG, EEG).

Since many of the deleterious effects of SB (dental wear, fractures of teeth and restorations, non-carious cervical dental lesions, tongue indentations, linea alba) are permanent, clinically speaking it is sometimes difficult to ascertain whether the patient has SB at the present time or whether the signs are sequelae of past SB (16).

When a patient seeks advice for a possible problem of $\mathrm{SB}$, diagnosis must begin with the patient's filling out a questionnaire containing a series of questions to orient the practitioner in the diagnostic process (20). Table 2 contains an example of some such questions. Nevertheless, it must be borne in mind that questionnaires have certain limitations, given the fluctuating course of SB (which causes errors of under- or overestimation in replies (21)) and the fact that the person who perceives the SB is frequently the bed partner, and because many

Table 1. Most-frequent Symptoms and Signs of SB.

\begin{tabular}{|rl|}
\hline Symptoms \\
\hline- & Grinding of teeth, accompanied by a characteristic sound that \\
& may even awaken the bruxer's bed partner. \\
\hline- & Pain in the TMJ. \\
\hline- & Pain in the masticatory and cervical muscles. \\
\hline- & Headache (especially in the temporal zone when the patient \\
& wakes up in the morning). \\
\hline- & Hypersensitive teeth. \\
\hline- & Excessive tooth mobility. \\
\hline- & Poor sleep quality. Tiredness. \\
\hline Signs & \\
\hline- & Abnormal tooth wear. \\
\hline- & Tongue indentations. \\
\hline- & Linea alba along the biting plane. \\
\hline- & Gum recession. \\
\hline- & Presence of torus maxillaris and/or mandibularis. \\
\hline- & Increase in muscle activity (This is recorded by the polysom- \\
\hline & nograph). \\
\hline- & Hypertrophy of masseter muscles. \\
\hline- & Reduction of salivary flow. \\
\hline- & Breakage of fillings and/or teeth. \\
\hline- & Limitation of mouth-opening ability. \\
\hline
\end{tabular}


Table 2. Examples of Questions That Can Help Diagnose SB.

\begin{tabular}{|c|c|}
\hline- & $\begin{array}{l}\text { Are you aware of frequently or occasionally clenching or grinding your } \\
\text { teeth while you are asleep? }\end{array}$ \\
\hline- & Has anyone ever told you you grind your teeth while you are asleep? \\
\hline- & $\begin{array}{l}\text { Do you notice tension or fatigue in your facial muscles, your teeth or } \\
\text { your gums when you wake up? }\end{array}$ \\
\hline- & $\begin{array}{l}\text { Have you ever awakened to find your jaw locked and you could not } \\
\text { open it? }\end{array}$ \\
\hline- & $\begin{array}{l}\text { Do you wake up to a headache, back pain or pain in the back of your } \\
\text { neck? }\end{array}$ \\
\hline- & Have you noticed wear on your teeth? \\
\hline
\end{tabular}

Table 3. PSG Criteria for Diagnosing SB.

\begin{tabular}{|ll|}
\hline - & At least two episodes of bruxism with audible sounds \\
\hline - & More than four episodes of bruxism per hour of sleep and/or \\
\hline - & More than 25 bursts of bruxism per hour of sleep and/or \\
\hline - & More than six bursts of rhythmic masticatory muscle activity per \\
& episode of bruxism
\end{tabular}

episodes of bruxism involve only the clenching of teeth without grinding and therefore are soundless.

In addition to these questionnaires, the AASM proposes a series of criteria for the clinical diagnosis of SB:

- The patient reports clenching or grinding of teeth during sleep

- One or more of the following clinical signs is observed:

o Abnormal wear on teeth

o Discomfort, fatigue or pain in the masticatory musculature and/or locking of the jaws on wakening

o Hypertrophy of the masseter muscles in forced voluntary clenching

o Masticatory muscle activity cannot be explained by any other sleep disorder, medical or neurological pathological process, ingestion of medications or any other type of medicinal product or drug.

Clinically, dental wear is the first sign to indicate the presence of bruxism, although it does not give any information about whether the bruxism is currently present or the wear is a sign of previous lesions. Furthermore, factors such as age, gender, occlusion, enamel hardness, diet, ingestion of acidic, isotonic or carbonic beverages, salivary flow and the presence of certain digestive disorders (gastroesophageal reflux, which often presents subclinically) have a very important influence on the variability of dental wear.

Other clinical signs of SB are listed in table 1.
The diagnostic devices available at present are more useful in research projects than in daily clinical use. Their high cost aside, the variability of presentation of SB (21) plus the artefacts and the annoyance caused by their application (wires, sensors adhered to the skin, sleeping in an unfamiliar environment) mean that sometimes these tests fail to have the desired validity. Diagnosis therefore continues to be based fundamentally on the clinical judgment of the practitioner.

Sleep bruxism has been analysed using intraoral devices that employ sensors to quantify clenching force and times of presentation. Sleep bruxism has also been analysed by observing the presence, distribution and progression of facets of wear in orthotic devices.

Portable electromyography (EMG) monitoring systems are another type of devices that can help in the diagnosis of SB. These have the advantage of moderate cost and the possibility of making multiple recordings in the patient's regular sleeping environment (22). Nevertheless, their validity has not yet been scientifically demonstrated in broad population groups. Because there is no audio/video record, ambulatory EMG devices tend to overestimate the presence of SB, as there are many motor activities (sighing, coughing, sucking) that may be confused with bruxism (Up to $30 \%$ of the masticatory muscle activity during sleep is not SB).

The only way to diagnose the presence of current SB with absolute certainty is by PSG in a sleep laboratory 
and confirmation that the patient meets the requirements described in the literature and listed in table 3.

A PSG test includes EEG recordings to measure brain activity, EMG recordings for masticatory muscle (masseter and temporal) activity, EKG recordings for heart activity, oximetry readings to measure blood oxygen levels and measurers of respiratory volume and frequency, in addition to audio/video recordings to rule out non-bruxing masticatory activity.

The greatest disadvantages of PSG are its high cost and its modification of the patient's habitual sleeping environment, which means that sometimes the patient's normal activity cycle is not reflected. For these reasons PSG is usually restricted to the diagnosis of complex cases (epilepsy, complex disorders of movement during sleep) and research purposes.

\section{Treatment}

At present there is no treatment that effectively, permanently eliminates the bruxing habit (23). For that reason the therapeutic approach to SB for now is oriented toward palliating, albeit partially, the effect of SB and preventing and treating its pathological effects on the stomatognathic apparatus (24).

One of the most important therapeutic tools is to give the patient information and a detailed, simple explanation of the clinical picture. Although the etiopathogenesis and physiopathology of SB seem to rest on central mechanisms that are beyond voluntary control (neurotransmitter activity, micro-arousals), the patient's cooperation in observation of the habit during wakefulness and the patient's engagement in the self-management of the habit through self-relaxation measures are very important elements for helping to reduce the frequency and intensity of masticatory muscle activity during wakefulness, which favours muscle relaxation and the reduction of bruxing episodes during sleep. If the patient does not assume responsibility for this important aspect of self-management in therapy and apply it, any other measures will be of only very limited usefulness. There are some pharmacological compounds (botulinum toxin type A, benzodiazepines and other muscle relaxants, anticonvulsants (25), beta blockers (15), dopamine and other dopaminergic drugs (13), antidepressants $(26,27)$, clonidine (15), etc.) that can help control SB, although their use must be restricted to nonrecurrent situations, such as the start of treatment or periods of exacerbation due to a rise in emotional tension, and always as part of a comprehensive, interdisciplinary approach.

Orthopaedic devices (oral orthotic devices) have not demonstrated their medium- and long-term usefulness in reducing masticatory muscle activity during sleep (28-31). Therefore at present they are indicated only for some clinical situations in which SB presents with painful muscular symptomatology and for the prevention of the pathological effects of parafunctional occlusal forces on teeth, the periodontium and odontological restorations.

Whereas it is fully proved that there is no relationship between the scheme of static and dynamic occlusal relations and the etiopathogenesis and physiopathology of SB (6), current scientific evidence does not support the use of irreversible occlusal treatments (occlusal adjustment through selective grinding, occlusal rehabilitation, orthodontics) for the treatment of SB.

Psychological therapies oriented toward controlling the psycho-emotional factors that may underlie SB (anxiety, emotional distress), such as behavioural cognitive therapy, EMG biofeedback, relaxation techniques, psychoanalysis, hypnosis and meditation (32-34), offer some favourable results, although their efficacy is still pending validation through studies of a high enough scientific caliber.

One very important aspect of the therapeutic handling of SB is the study and treatment of sleep disorders, since improvement in the quantity and quality of sleep entails an important reduction in episodes of bruxism.

Some types of physical medicine (cardiovascular toning programmes, TENS, acupuncture, manual massage) (35-39) and alternative/naturopathic medicine (40) are also employed.

\section{Conclusions}

Sleep bruxism is a parafunctional oromotor habit with a high prevalence in the general population. Because of its clinical implications, it has been and continues to be a point on which great attention is focused by odontology.

Throughout history there have been series of different hypotheses posited to explain the etiopathogenesis and physiopathology of SB. At present a multi-factor etiological model is accepted that includes genetic, neurophysiological (central neurotransmitters, sleep structure, autonomic nervous system), psycho-emotional and pharmacological factors.

Contrary to general belief, only a small percentage of bruxers develop a pathological picture as a consequence of the SB habit. This occurs in the cases where SB oversteps the body's capacity to adapt, in which event SB may cause craniomandibular dysfunction.

At present there is no effective treatment to eliminate SB permanently. Therefore the therapeutic approach is steered toward attempting to prevent damage and treating the pathological effects SB has on the structures of the masticatory system. 


\section{References}

References with links to Crossref - DOI

1. Kato T, Thie NM, Huynh N, Miyawaki S, Lavigne GJ. Topical review: sleep bruxism and the role of peripheral sensory influences. J Orofac Pain. 2003;17:191-213.

2. Lavigne GJ, Montplaisir JY. Restless legs syndrome and sleep bruxism: prevalence and association among Canadians. Sleep. 1994;17:739-43.

3. Abe K, Shimakawa M. Genetic and developmental aspects of sleeptalking and teeth-grinding. Acta Paedopsychiatr. 1966;33:33944.

4. Hublin C, Kaprio J, Partinen M, Koskenvuo M. Sleep bruxism based on self-report in a nationwide twin cohort. J Sleep Res. 1998;7:61-7.

5. Okeson JP, Phillips BA, Berry DT, Cook Y, Paesani D, Galante J. Nocturnal bruxing events in healthy geriatric subjects. J Oral Rehabil. 1990;17:411-8.

6. Vanderas AP, Manetas KJ. Relationship between malocclusion and bruxism in children and adolescents: a review. Pediatr Dent. 1995; 17:7-12.

7. Lavigne GJ, Rompré PH, Guitard F, Sessle BJ, Kato T, Montplaisir JY. Lower number of K-complexes and K-alphas in sleep bruxism: a controlled quantitative study. Clin Neurophysiol. 2002;113:686-93.

8. Schneider C, Schaefer R, Ommerborn MA, Giraki M, Goertz A, Raab WH, et al. Maladaptive coping strategies in patients with bruxism compared to non-bruxing controls. Int J Behav Med. 2007;14:257-61.

9. Lobbezoo F, Lavigne GJ, Tanguay R, Montplaisir JY. The effect of catecholamine precursor L-dopa on sleep bruxism: a controlled clinical trial. Mov Disord. 1997;12:73-8.

10. Chen WH, Lu YC, Lui CC, Liu JS. A proposed mechanism for diurnal/nocturnal bruxism: hypersensitivity of presynaptic dopamine receptors in the frontal lobe. J Clin Neurosci. 2005;12:161-3.

11. Lavigne GJ, Khoury S, Abe S, Yamaguchi T, Raphael K. Bruxism physiology and pathology: an overview for clinicians. J Oral Rehabil. 2008;35:476-94.

12. Macaluso GM, Guerra P, Di Giovanni G, Boselli M, Parrino L, Terzano MG. Sleep bruxism is a disorder related to periodic arousals during sleep. J Dent Res. 1998;77:565-73.

13. Lavigne GJ, Soucy JP, Lobbezoo F, Manzini C, Blanchet PJ, Montplaisir JY. Double-blind, crossover, placebo-controlled trial of bromocriptine in patients with sleep bruxism. Clin Neuropharmacol. 2001;24:145-9.

14. Kato T, Rompré P, Montplaisir JY, Sessle BJ, Lavigne GJ. Sleep bruxism: an oromotor activity secondary to micro-arousal. J Dent Res. 2001;80:1940-4.

15. Huynh N, Lavigne GJ, Lanfranchi PA, Montplaisir JY, de Champlain J. The effect of 2 sympatholytic medications--propranolol and clonidine-on sleep bruxism: experimental randomized controlled studies. Sleep. 2006;29:307-16.

16. Lavigne GJ, Rompré PH, Montplaisir JY. Sleep bruxism: validity of clinical research diagnostic criteria in a controlled polysomnographic study. J Dent Res. 1996;75:546-52.

17. Kato T, Thie NM, Montplaisir JY, Lavigne GJ. Bruxism and orofacial movements during sleep. Dent Clin North Am. 2001;45:65784.

18. De Laat A, Macaluso GM. Sleep bruxism as a motor disorder. Mov Disord. 2002;17 Suppl 2:S67-9.

19. Lobbezoo F, Lavigne GJ. Do bruxism and temporomandibular disorders have a cause-and-effect relationship? J Orofac Pain. 1997;11:15-23.

20. Carlsson GE, Egermark I, Magnusson T. Predictors of bruxism, other oral parafunctions, and tooth wear over a 20 -year follow-up period. J Orofac Pain. 2003;17:50-7.

21. Lavigne GJ, Guitard F, Rompré PH, Montplaisir JY. Variability in sleep bruxism activity over time. J Sleep Res. 2001;10:237-44.

22. Harada T, Ichiki R, Tsukiyama Y, Koyano K. The effect of oral splint devices on sleep bruxism: a 6-week observation with an ambulatory electromyographic recording device. J Oral Rehabil. 2006;33:482-8.

23. Huynh N, Manzini C, Rompré PH, Lavigne GJ. Weighing the potential effectiveness of various treatments for sleep bruxism. J Can Dent Assoc. 2007;73:727-30.

24. Huynh NT, Rompré PH, Montplaisir JY, Manzini C, Okura K, Lavigne GJ. Comparison of various treatments for sleep bruxism using determinants of number needed to treat and effect size. Int $\mathbf{J}$ Prosthodont. 2006;19:435-41.

25. Lobbezoo F, Van der Zaag J, Van Selms MK, Hamburger HL, Naeije M. Principles for the management of bruxism. J Oral Rehabil. 2008;35:509-23.

26. Mohamed SE, Christensen LV, Penchas J. A randomized doubleblind clinical trial of the effect of amitriptyline on nocturnal masseteric motor activity (sleep bruxism). Cranio. 1997;15:326-32.

27. Raigrodski AJ, Mohamed SE, Gardiner DM. The effect of amitriptyline on pain intensity and perception of stress in bruxers. J Prosthodont. 2001;10:73-7.

28. Macedo CR, Silva AB, Machado MA, Saconato H, Prado GF. Occlusal splints for treating sleep bruxism (tooth grinding). Cochrane Database Syst Rev. 2007;4:CD005514.

29. Landry ML, Rompré PH, Manzini C, Guitard F, de Grandmont P, Lavigne GJ. Reduction of sleep bruxism using a mandibular advancement device: an experimental controlled study. Int J Prosthodont. 2006;19:549-56.

30. Van der Zaag J, Lobbezoo F, Wicks DJ, Visscher CM, Hamburger HL, Naeije M. Controlled assessment of the efficacy of occlusal stabilization splints on sleep bruxism. J Orofac Pain. 2005;19:151-8.

31. Klasser GD, Greene CS. Oral appliances in the management of temporomandibular disorders. Oral Surg Oral Med Oral Pathol Oral Radiol Endod. 2009;107:212-23.

32. Glaros AG, Burton E. Parafunctional clenching, pain, and effort in temporomandibular disorders. J Behav Med. 2004;27:91-100.

33. Ommerborn MA, Schneider C, Giraki M, Schäfer R, Handschel J, Franz M, et al. Effects of an occlusal splint compared with cognitive-behavioral treatment on sleep bruxism activity. Eur J Oral Sci. 2007;115:7-14.

34. Winocur E, Gavish A, Emodi-Perlman A, Halachmi M, Eli I. Hypnorelaxation as treatment for myofascial pain disorder: a comparative study. Oral Surg Oral Med Oral Pathol Oral Radiol Endod. 2002;93:429-34.

35. Ackerman JB. A new approach to the treatment of bruxism and bruxomania. N Y State Dent J. 1966;32:259-61.

36. Knutson GA. Vectored upper cervical manipulation for chronic sleep bruxism, headache, and cervical spine pain in a child. J Manipulative Physiol Ther. 2003;26:E16.

37. Jardini RS, Ruiz LS, Moysés MA. Electromyographic analysis of the masseter and buccinator muscles with the pro-fono facial exerciser use in bruxers. Cranio. 2006;24:29-37.

38. Alvarez-Arenal A, Junquera LM, Fernandez JP, Gonzalez I, Olay S. Effect of occlusal splint and transcutaneous electric nerve stimulation on the signs and symptoms of temporomandibular disorders in patients with bruxism. J Oral Rehabil. 2002;29:858-63.

39. Jadidi F, Castrillon E, Svensson P. Effect of conditioning electrical stimuli on temporalis electromyographic activity during sleep. J Oral Rehabil. 2008;35:171-83.

40. Etzel KR, Stockstill JW, Rugh JD, Fisher JG. Tryptophan supplementation for nocturnal bruxism: report of negative results. J Craniomandib Disord. 1991;5:115-20. 